\title{
El Pensamiento Crítico y Reflexivo desde la Percepción de los Estudiantes de la Licenciatura en Enfermería
}

\author{
Gloria Margarita Ruiz Gómez, Dra. \\ Manuel Antonio López Cisneros, Dr. \\ Juan Yovani Telumbre Terrero, Mtro. \\ Alma Delia Sánchez Rivero, Dra. \\ Karen Doribel López Hernández, Lic. \\ Universidad Autónoma del Carmen, México
}

Doi: 10.19044/esj.2018.v14n27p102 URL:http://dx.doi.org/10.19044/esj.2018.v14n27p102

\begin{abstract}
The nursing profession has gone through a process of professionalization in the last decades, which allows to recognize that Nursing emerged as a social activity that has been institutionalized, both in its training and in its work practice, affirming itself as a science, profession, discipline and art. Nursing professionals must generate, promote and apply reflective and critical thinking in their actions, professional, ethical, humanistic and technological (Cardenas, 2015). The objective of this study is to describe the reflexive and critical thinking of the students of the Degree in Nursing of the Faculty of Health Sciences. This is a qualitative study, carried out with 5 students of the faculty of health sciences, we used a representative sample of students who are attending the 2nd, 4th and 8th semester of Nursing Degree. Data were collected through a semi-structured interview (Face to Face). Results: From the constant comparison of data analysis emerged the subcategories 1) critical thinking; 2) reflective thinking; 3 ) decision making; 4) reflective practice. Conclusions: The results of this study achieved the objective of describing reflective thinking and critical thinking in students of the Nursing Degree in the Faculty of Health Sciences. We identified the empirical categories of the study phenomenon that represents the result attributed to the perception of Critical and Reflective thinking. This resulted in four interrelated sub-categories, reflective thinking, critical thinking, decision-making and reflective practice.
\end{abstract}

Keywords: Critical and Reflective Thinking, perception, nursing. 


\section{Resumen}

La profesión de enfermería ha transitado por un proceso de profesionalización en las últimas décadas, lo cual permite reconocer que Enfermería surgió como una actividad social que se ha institucionalizado, tanto en su formación como en su práctica laboral, afirmándose como ciencia, profesión, disciplina y arte. Los profesionales de enfermería deben de generar, promover y aplicar el pensamiento reflexivo y crítico en sus acciones, profesionales, éticas, humanísticas y tecnológicas (Cárdenas, 2015). El Objetivo de este estudio es describir el pensamiento reflexivo y crítico de los estudiantes de la Licenciatura en Enfermería de la Facultad de Ciencias de la Salud. Se trata de un estudio cualitativo, realizado con 5 estudiantes de la facultad de ciencias de la salud, se utilizó una muestra representativa de alumnos que están cursando el $2^{\text {do }}$, el $4^{\text {to }}$ y $8^{\text {vo }}$ semestre de Licenciatura en Enfermería. Los datos se recolectaron a través de una entrevista semiestructurada (Face to Face). Resultados: De la comparación constante del análisis de datos emergieron la subcategorías 1)pensamiento crítico; 2) pensamiento reflexivo; 3) toma de decisiones; 4) práctica reflexiva. Conclusiones: Los resultados de este estudio lograron el objetivo, el de describir el pensamiento reflexivo y pensamiento crítico en los estudiantes de la Licenciatura en Enfermería en la Facultad de Ciencias de la Salud. Se identificaron las categorías empíricas del fenómeno de estudio que representa al resultado atribuido a la percepción del pensamiento Crítico y Reflexivo. Lo que dio como resultado cuatro subcategorías, que se relacionan entre sí, el pensamiento reflexivo, el pensamiento crítico, la toma de decisiones y la práctica reflexiva.

Palabras Claves: Pensamiento critico y reflexivo, percepción, enfermería.

\section{Introducción}

El aprendizaje es algo que se puede ver como un proceso en el cual el individuo va obteniendo habilidades, destrezas, aptitudes, actitudes y compromiso social, es aquí donde la reflexividad constituye como punto de partida indispensable para el diseño de estrategias y metodologías que guíen el desarrollo profesional, académico y de investigación (Pacheco, 2009).

Se destaca la necesidad de explorar los factores que influyen en los alumnos a no desarrollar el pensamiento crítico y reflexivo, así como las limitaciones que presentan las actuales prácticas de enseñanza-aprendizaje, en las cuales predomina la acumulación de información por sobre al ánimo de promover una verdadera capacidad de pensar.

Según Durgahe (1998) menciona que el potencial de la reflexión como método de aprendizaje y de desarrollo intelectual, ha sido descrito en 
diversas investigaciones, que sostienen que la reflexión ayuda a contextualizar los cuidados enfermeros a integrar la teoría y la práctica. Utilizada con eficacia, la reflexión puede contestar a preguntas sobre la naturaleza de la práctica enfermera y puede ayudar a generar teoría a partir de la práctica (Cárdenas, 2014).

El pensamiento es la actividad y creación de la mente, es un proceso complejo, propio de los seres humanos el cual le da la capacidad para ordenar, el pensamiento como actividad mental puede ser libre y espontánea, no puede hablarse del pensamiento crítico y reflexivo, sin tener en cuenta el propósito y la autodirección de lo que se piensa (Cárdenas, 2014).

Según González, (2006) para poder desarrollar un pensamiento crítico se debe adoptar una buena aptitud, mantener una mente abierta, libertad de pensamiento, humildad intelectual, estos son los principales factores en el cual los alumnos carecen, para poder desarrollar un buen pensamiento crítico.

En la Licenciatura en Enfermería de la Facultad de Ciencias de la Salud de la Universidad Autónoma del Carmen (UNACAR) se implemento el modelo educativo por competencias que constituye un método de vanguardia, donde se integran nuevas actitudes, habilidades, conocimiento y relaciones sociales, y también nuevas estrategias para evidenciar los saberes adquiridos, aprendizaje para la solución de problemas y la transformación de la realidad; construir y reconstruir el conocimiento, aprender y desaprender; aprendizajes basado en problemas, orientando a proyectos, estudios de casos, evidencias y trabajo colaborativo. Ya que este modelo basado en competencia es un modelo socio formativo, trata de las condiciones educativas esenciales para facilitar la educación por competencia, se caracteriza por un aprendizaje centrado en el estudiante permitiendo que el estudiante pase de un nivel principiante a experto, como lo describe Patricia Benner.

Identificar como realiza el estudiante este proceso: de tal forma que sea el alumno quien identifique sus debilidades y necesidades a través del pensamiento reflexivo ellos desarrollan herramientas que les permite convertirse en aprendices más efectivo. Los estudiantes necesitan examinar su trabajo y pensar sobre lo que hace bien y cuáles son las áreas en las que aún necesitan ayuda.

Según Díaz, (2006) Menciona que existen procedimientos más eficaces para implicar a los estudiantes en su proceso de aprendizaje a los que le denomina buenas practicas docentes. En este sentido se ha señalado que los docentes son los responsables de la formación y complementación de currículos que formen y egresen profesionales capaces de desarrollar la capacidad del pensamiento reflexivo y crítico, ya que el alumno es el eje central en el proceso de aprendizaje - enseñanza, por lo tanto se necesita que el docente sea capaz de desarrollar métodos estratégicos y estimular al estudiante para que desarrolle ideas propias o crear independencia intelectual, 
bajo este modelo el docente deja de ser un transmisor de conocimientos para convertirse en un educador real (guía o referente) que influye en las nuevas generaciones (mediador del conocimiento).

Bajo estas concepciones en la Facultad de Ciencias de la Salud de la UNACAR es necesario contar con la implementación de un pensamiento reflexivo y crítico en los estudiantes de enfermería para formar enfermeras(os) profesionales con la más alta calidad que vaya acorde con los avances científicos y tecnológicos de la Educación Superior en el contexto nacional e internacional. En esta línea el propósito de esta investigación es conocer la percepción del pensamiento reflexivo y crítico en los estudiantes de la licenciatura de enfermería de la Universidad Autónoma del Carmen, esto con la finalidad de generar la competencia del pensamiento crítico y reflexivo del estudiante para que el alumno sea capaz de enfrentar o resolver situaciones complejas.

Llobet (2013) menciona que en la actualidad el concepto de pensamiento crítico se ha utilizado en diferentes medios, el pensamiento es una competencia humana, la descripción etimológica la refiere como la facultad o capacidad para pensar. Más sin embargo Becerril define que el pensamiento crítico es un proceso complejo que puede definirse de múltiples formas, un sinónimo es razonamiento (Cárdenas, 2005).

Alcorta E. (2008) menciona que el pensamiento crítico es el proceso intelectual que en forma decidida y autorregulada busca llegar a juicios razonables, es decir se puede entender como la forma de pensar en la que se ejerce esta acción y se mejora la calidad de su pensamiento. En el sentido de que está bien definido el pensamiento, lo que implica claridad, exactitud, precisión, pertinencia, profundidad, amplitud y lógica.

Díaz-Montenegro, (2009) El pensamiento crítico es un pensamiento fundamentalmente razonable; no es un pensamiento fortuito o arbitrario. Por lo contrario, constituye un proceso cognitivo complejo de pensamiento que reconoce el predominio de la razón sobre las otras dimensiones del pensamiento. El pensamiento crítico es razonable en cuanto que va en busca de la verdad. Su finalidad es reconocer aquello que es justo y aquello que es verdadero.

Marie, De la Garza, Slade, Fortune, Pallasio \& Mongeau, (2003) realizaron un estudio basado en la teoría de Lipman donde afirma que el pensamiento crítico "nos protege contra el hecho de creer de manera forzosa lo que nos dicen los demás, sin que tengamos la oportunidad de investigar por nosotros mismos". Ayuda, así, a las personas a pensar mejor y a elaborar mejores juicios.

El pensamiento reflexivo es una consideración activa, persistente y cuidadosa de una creencia, a partir de los fundamentos que la sustentan y de las posteriores conclusiones (Romero, 2007). 
González, (2012) El pensamiento reflexivo permite la transformación de las prácticas y contribuye a resinificar la cultura para responder a las necesidades de los individuos; y de esta forma ir progresivamente encontrando los valores que componen una auténtica educación en cada contexto particular como valor humano en proceso de descubrimiento.

Las personas con un buen nivel reflexivo y pensamiento crítico son descritos como personas de mente abiertas, flexibles, indagadoras, comunicativas, asertivas, responsables y autónomas en su aprendizaje. Por eso el pensamiento reflexivo y crítico es ese modo de pensar sobre cualquier tema, contenido o problema, en el cual el estudiante mejora la calidad de su pensamiento.

El pensamiento reflexivo y crítico en enfermería es una herramienta básica y fundamental para el estudiante y futuro profesional porque le ayuda a desarrollar las relaciones con los pacientes, ya que a pesar de que existen abundancia literaturas de reflexión aún no tienen claramente el concepto, y de ahí viene la dificultad el uso que hacen las enfermeras(os) en sus prácticas. Este desorden conceptual referido a los procesos cognitivos asociados a la reflexión puede dar lugar a un qué los profesionales crean que están reflexionando cuando en realidad no es así (Burton, 2000).

García, (2001) menciona lo importante que es el Modelo Educativo ya que identifica a una institución, le da personalidad esta misma se plantea a los alumnos, quienes se empiezan a identificar y empieza a transcender en sus vidas adoptando su propia identidad como alumno y persona. El modelo educativo de igual manera son visiones de teorías o pedagógicas que orienta a las especialistas y a los profesores es de gran ayuda, en el proceso de enseñanza-aprendizaje, o bien en la compresión del programa de estudios.

En la Universidad Autónoma del Carmen se ha actualizado en el desarrollo de enseñanza y aprendizaje, implementando el modelo educativo basado en competencias, centrándolo en el aprendizaje del alumno en apego a la realidad. Esta transición ha abierto diversas oportunidades para generar prácticas educativas innovadoras al interior de los programas de estudios, en especial aquellos cursos que permean en forma trasversal en la currícula y que influyen en la toma de decisiones de otras.

El Modelo Educativo Acalan está orientado hacia las habilidades de razonamiento y cuestionamiento, al desarrollo de competencias para solucionar problemas reales de la sociedad. Es por ello, que los docentes diseñan los ambientes de aprendizaje, así como las actividades, los criterios de evaluación, sin descuidar los materiales y recursos didácticos, así como lo relacionado al desarrollo del pensamiento reflexivo y crítico, Ya que es de suma necesidad en la independencia en enfermería. La educación basada en competencia es un enfoque sistemático del conocer y del desarrollo de habilidades, y se determina a partir de funciones y tareas precisas. 


\section{Metodología}

Se trata de una investigación cualitativa con la participación de entrevistas personales (face to face) de estudiantes del 2do, ${ }^{4 t o}$ y 8to semestre de la licenciatura de Enfermería de la Facultad de Ciencias de la Salud.

Es un estudio descriptivo-exploratorio y retrospectivo, con enfoque constructivista en el cual se realiza una crítica argumentando sobre el pensamiento reflexivo y crítico de los estudiantes de la licenciatura de enfermería; con la finalidad de conocer una visión general y de aproximación sobre las prácticas reflexivas y el pensamiento crítico de los estudiantes durante su formación profesional. En segundo punto se realiza a nivel desertivo mediante la realización de técnicas e instrumentos para la recolección de los datos que puedan enfocarse en el levantamiento bibliográfico y documental o en entrevista y/o observaciones. Se realiza entrevistas semi-estructurada sobre la práctica reflexiva y el pensamiento crítico para la recolección de datos con preguntas enfocadas sistematizadas que nos permitan tener una aproximación de los antecedentes del objeto de estudio (estudiantes).

La población estuvo representada por los estudiantes del programa educativo de Licenciatura en Enfermería y se utilizó una muestra representativa de alumnos que están cursando el $2^{\text {do }}$, el $4^{\text {to }}$ y $8^{\text {vo }}$ semestre de Licenciatura en Enfermería. Se realizó entrevista personal (face to face), a los participantes de la investigación. Los cuales firmaron el consentimiento informado, posteriormente a que se les realizará la entrevista personal que constaba de una cedula de datos generales, y cinco preguntas abiertas y a profundidad, dirigidas a los estudiantes de Licenciatura en Enfermería, que participaban en el estudio de investigación.

La entrevista face to face que se le realizó a los estudiantes, permitió reconocer y plantear situaciones, cuantificar aspectos de la realidad, identificar las formas y relaciones que se presentan en la vida cotidiana de igual manera brindar de los servicios profesional de la salud y favorecer el desarrollo y progreso del cuidado de enfermería.

Las entrevistas fueron grabadas y posteriormente se transcribieron de tal forma que se realizó un análisis comparativo cíclico hasta la saturación de datos, también se realizó la revisión de la literatura para la recolección de la información de las categorizaciones. Las entrevistas personalizadas (face to face), concluyeron con la saturación de los datos a los entrevistados (estudiantes), cada entrevista será capturada y codificada al término de cada intervención, se categorizaran y codificaran los hallazgos y contructos.

Se realizara codificación abierta mediante separación, comparación y conceptualización del pensamiento crítico reflexivo de los estudiantes, se separaran en líneas, oraciones o por párrafos y al final serán categorizados y comparados por similitudes y diferencias de tal forma que se extraigan los 
pensamientos e ideas y significados de los estudiantes referente al pensamiento crítico y reflexivo.

Para el análisis de los hallazgos, se categorizaran por comparativos, inicialmente se realizara una comparación inadente con categoría de categoría y de tal forma que se determinen los constructos, distinguiendo las similitudes entre el pensamiento crítico y reflexivo de los estudiantes de la Licenciatura en Enfermería.

Se trata de una investigación cualitativa con participación de grupos focales de $1^{\text {er }}$ y $9^{\text {no }}$ semestre de la licenciatura de Enfermería de la Facultad de Ciencias de la Salud. El Estudio es de tipo descriptivo-exploratorio y retrospectivo, constructivismo en el cual se realizara una crítica argumentando sobre el pensamiento reflexivo y crítico de los estudiantes de la licenciatura de enfermería; con la finalidad de conocer una visión general y de aproximación sobre las practicas reflexivas y el pensamiento crítico de los estudiantes durante su formación profesional.

Se realizaron entrevistas estructuradas sobre la práctica reflexiva y el pensamiento crítico para la recolección de datos con preguntas enfocadas sistematizadas que nos permitan tener una a aproximación a los antecedentes del objeto de estudios (estudiantes). Se empleó el programa SPSS 18.0. Se utilizó estadística descriptiva, con medidas de frecuencia, dispersión y tendencia central. La población del estudio estará conformada por estudiantes de la Licenciatura en enfermería de la facultad de ciencias de la salud de la UNACAR.

Se aplicó un cuestionario de elaboración propia, donde se recolectaron datos de las prácticas docentes dirigidas al desarrollo de la competencia de pensamiento reflexivo-critico de los estudiantes. Se aplicó un cuestionario de elaboración propia dirigido a directivos responsables del curriculum de enfermería, para identificar la relevancia de la competencia de pensamiento reflexivo-critico en el plan curricular.

Para el estudio se contó con la aprobación del Comité de Investigación de la facultad de Ciencias de la Salud, así como con la autorización de la institución de salud donde se llevaron a cabo el estudio, además del consentimiento informado de los estudiantes.

El rigor científico de la investigación radica en los criterios éticos (principio de beneficencia y respeto a la dignidad humana) y de audibilidad (credibilidad, confiabilidad, a través del consentimiento informado, audibilidad y transferibilidad).

\section{Resultados y Discusión}

En esta investigación participaron 5 alumnos de la Licenciatura en Enfermería de la Facultad de Ciencias de la Salud con estado civil soltera: dos 
de 19 años y en la actualidad cursando Segundo Semestre, una de 20 años de cuarto semestre y dos de 23 años de octavo semestre.

A continuacion se presenta la descripción de las categorías del fenómeno de estudios que representa los significados atribuidos. Para cuidar la identidad de las alumnas se le denomino (P1)para la participante numero uno, (P2) para la participante numero dos, (P3)para la participante numero tres, (P4)participante numero cuatro, (P5)participante numero cinco. Notesé la diferencia en las narraciones emitidas por las participantes referente a los significados.

\section{Categoria 1: pensamiento Crítico.}

...Es la manera de auto evaluarse referente a los proyectos que uno realiza en la vida diaria . $(\mathrm{P} 1)$

... Nos lleva a ver las cosas con más claridad las cosas.(P2)

... Es aquel que nosotros tenemos para cuestionar a otras personas, para cuestionar una situación, como llevarla como saber qué es lo que es, como entender aquella persona que está llevando una situación parecida.(P3)

... Seria cuestionarme algo como yo veo las cosas, no es como lo ve otra persona, a como lo veo yo, puedo decir que eso está mal y está bien para mi forma de verlo o como yo me expreso... (P4)

\section{Categoría 2: Pensamiento Reflexivo.}

... Para mí el pensamiento reflexivo es analizar alguna situación en la que esté involucrado uno y ver como se soluciona si es un problema... (P2)

... Nos ayuda a reflexionar sobre los avances y también sobre las deficiencias que tenemos como alumnos...(P3)

... En la cual nosotros nos basamos según nuestras ideas cada quien tiene una manera de pensar, entonces el momento de reflexionar una situación la va a ver de un punto de vista muy diferente, nos va ayudar a cómo salir, como arreglarla como solucionarla que riesgo tiene y cómo podemos evitar otra situación como esa... (P4)

\section{Categoría 3: Toma de decisiones.}

... Cuando nosotros iniciamos creo que no teníamos mucho que dar a conocer que criticar en este caso, pero dado al paso del tiempo durante las prácticas que hemos tenido, ahorita si hemos hecho nuestras evaluaciones en el pensamiento crítico y hemos plasmado lo que nosotros creemos en lo que nos han enseñado en el aula.(P1)

... Es algo que me ha estado moviendo mucho personal mente tiene eso de que había materias que si conozco a la perfección y hay materias que simplemente vengo en blanco pues simplemente me cuesta mucho trabajo adaptarme a las materias, pero me pongo a ver cuáles son mis herramientas, 
para entenderlas entonces empiezo a investigar y a investigar ya cuando entiendo bien la materia y de que se trata y en que se basa ahora si trato de ver que es lo que voy hacer durante el semestre llevar la materia con una buena calificación y no perderme... (P5)

\section{Categoría 4: Práctica reflexiva.}

... Para mí ha sido el saber el conocer y el poner en práctica todo lo aprendido en el aula.(P1)

... Ante una situación de algún paciente más bien sería algo muy rápido en la que tendría trabajar para no gastar mucho tiempo pensando cómo voy a curar, para que ella no tenga tantos contratiempos en su enfermedad y poder ayudarla a salir adelante.(P4)

En la (Ilustración 1) que se muestra a continuación pretende explicar la relación entre las categorías antes mencionadas.

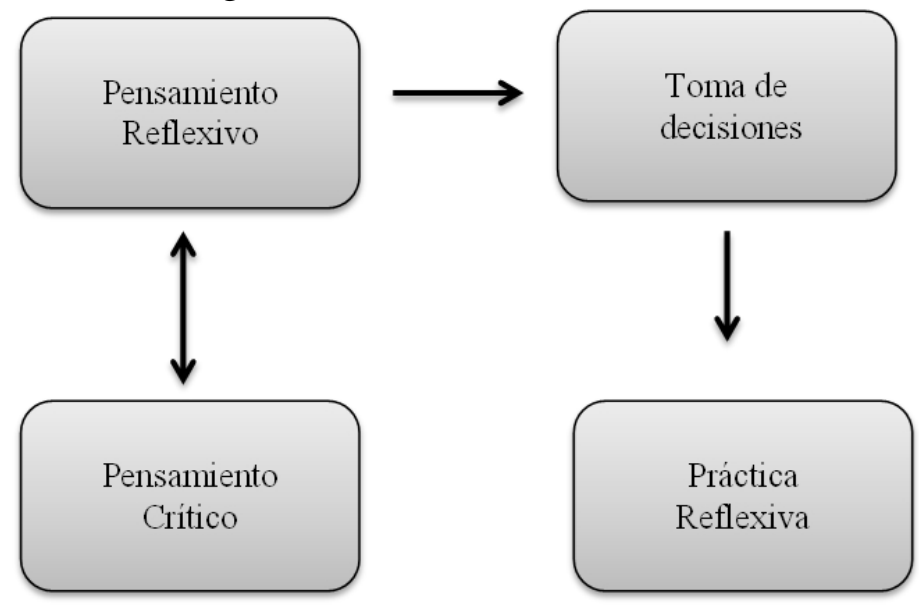

Ilustración 1. Relación de las categorías

\section{Discusión}

En este apartado, se presenta algunos fragmentos de la descripción de las categorías empíricas del fenómeno de estudio que representa al resultado atribuido a la percepción del pensamiento crítico y reflexivo.

\section{Categoría 1: pensamiento crítico}

El pensamiento crítico implica la reflexión y a veces la suspensión del juicio también implica la evaluación y valoración crítica su actividad es la capacidad de análisis e interpretación. El proceso del pensamiento crítico se orienta a hacer juicios sobre muchas situaciones se tropiece sobre una la vida diaria ( Profetto-McGrath,2005). Mota de Cabrera (2010), refiere que el pensamiento crítico involucra el desarrollo de una serie de habilidades que 
determinan cómo persuadir y ser persuadidos. Aunque parezca sencillo, este proceso involucra literalmente todo lo que sabemos acerca de nosotros mismos. Lo cual se relaciona con los resultados obtenidos de los estudiantes P1 y P2 que sustentan ...... que el pensamiento crítico.....

P1.....Es la manera de auto evaluarse referente a los proyectos que uno realiza en la vida diaria.

P2.....Nos lleva a ver las cosas con más claridad.

Los alumnos tienen algunas nociones de lo que es el pensamiento crítico; algunos piensan que es negativo, como hacer un juicio, o la capacidad de opinar o manifestar un punto de vista personal, sea o no fundamentado

(Paul, Binker, Martin, Vetrano y Kreklau,1995).

Los estudiantes de Licenciatura en Enfermería refieren...

P3 ...que el Pensamiento Crítico... Es aquel que nosotros tenemos para cuestionar a otras personas, para cuestionar una situación como llevarla, como saber qué es lo que es, como entender aquella persona que está llevando una situación parecida...

P4... Sería cuestionarme algo como yo veo las cosas, no es como lo ve otra persona, a como lo veo yo, puedo decir que es lo que está mal y está bien para mi forma de verlo o como yo me expreso...

\section{Categoría 2: pensamiento reflexivo}

El pensamiento reflexivo se entiende como la capacidad de reformular el propio pensamiento, mediante el uso del lenguaje, lo que lleva a reflexionar sobre el comportamiento de si mismo y las acciones de los demás, para facilitar la transformación del sentido mismo (González - Moreno , 2009). La cual se relaciona con los estudiantes denominado...

P2 ...Para mi el pensamiento reflexivo es analizar alguna situación en la que esté involucrado uno y ver como se soluciona si es un problema.

P4... En la cual nosotros nos basamos según nuestras ideas cada quien tiene una manera de pensar, entonces el momento de reflexionar una situación la va a ver desde un punto de vista muy diferente, nos va ayudar a cómo salir, como arreglarla como solucionarla que riesgo tiene y cómo podemos evitar otra situación como esa.

Buron-Ojedas (1995), refiere en los estudios realizados que no pocos alumnos llegan a cursos avanzados sin saber realizar tareas básicas para el aprendizaje, incluso en las universidad, hay estudiantes que muestran grandes dificultades para comprender un texto, para resumirlo y para juzgarlo, en la cual el alumno sustenta que el pensamiento reflexivo...

P3... Nos ayuda a reflexionar sobre los avances y también sobre las deficiencias que tenemos como alumnos. 


\section{Categoría 3: toma de decisiones}

La autonomía y la responsabilidad de un profesional no se entienden sin una gran capacidad de reflexionar en la acción y sobre la acción. Esta capacidad está en el interior del desarrollo permanente, según la propia experiencia, las competencias y los conocimientos profesionales de cada uno de los alumnos (Perrenoud, 2001). De tal manera que los alumnos a lo sustentado ellos relatan que....

P1 ... Cuando nosotros iniciamos creo que no teníamos mucho que dar a conocer que criticar en este caso, pero dado al paso del tiempo durante las prácticas que hemos tenido, ahorita si hemos hecho nuestras evaluaciones en el pensamiento crítico y hemos plasmado lo que nosotros creemos en lo que nos han enseñado en el aula.

P4 ...Es algo que me ha estado moviendo mucho personal mente tiene eso de que había materias que si conozco a la perfección y hay materias que simplemente vengo en blanco pues simplemente me cuesta mucho trabajo adaptarme a las materias, pero me pongo a ver cuáles son mis herramientas para entenderlas entonces empiezo a investigar y a investigar ya cuando entiendo bien la materia y de que se trata y en que se basa ahora si trato de ver que es lo que voy hacer durante el semestre llevar la materia con una buena calificación y no perderme.

P5 ...Poder saber cómo la voy a llevar acabo, más que nada emprender una nueva meta para poder alcanzar y empezar a cuestionarme como la voy a solucionar que es lo que tengo que tener.

\section{Categoría 4: práctica reflexiva}

Johns y de agua dulce (1998), también describen el valor de la práctica reflexiva como medio de aprendizaje. No hay duda de que la "reflexión" es un concepto complejo que ha desafiado el consenso sobre la definición, aunque existen algunos puntos en común.

Boud, et al (1985), hace mención sobre que la reflexión es un foro de respuestas del alumno a experimentar.

Jarvis(1992), hace mención que la practica reflexiva no solo es practica si no es ese tipo de práctica que busca problematizar muchas situaciones de desempeño profesional para que puedan convertirse en posibles situaciones de aprendizaje y por lo que los profesionales puedan seguir aprendiendo, creciendo y desarrollándose relacionado con lo dicho p1 y p4 mencionan...

P1 ...Para mí ha sido el saber el conocer y el poner en práctica todo lo aprendido en el aula .

P4 ...Ante una situación de algún paciente más bien sería algo muy rápido en la que tendría que trabajar para no gastar mucho tiempo pensando 
cómo voy a curar, para que ella no tenga tantos contratiempos en su enfermedad y poder ayudarla a salir adelante.

\section{Conclusión}

Los resultados de este estudio lograron el objetivo, el de describir el pensamiento reflexivo y pensamiento crítico en los estudiantes de la Licenciatura de Enfermería en la Facultad de Ciencias de la Salud. Durante las entrevistas del estudio de investigación, se identificaron cuatro subcategorías, que se relacionan entre sí, el pensamiento reflexivo, el pensamiento crítico, la toma de decisiones y la práctica reflexiva. Estas subcategorías fueron identificadas en los resultados de las preguntas que se le hicieron a los participantes de la investigación, y las cuales han sido sustentadas con estudios relacionados con cada subcategoría. Esto quiere decir que los estudiantes de la Licenciatura en Enfermería, deben de transitar durante su formación profesional de un pensamiento critico y reflexivo, hacia la toma de decisiones y la práctica reflexiva, lo cual permitirá que el futuro profesional desempeñe sus funciones sustantivas, como un profesional con práctica reflexiva: con un paradigma integrador y abierto.

Que con lleve a una verdadera práctica reflexiva, de manera permanente con una relación analítica continua con la acción, de tal forma que se convierta en algo relativamente independiente de los obstáculos que aparezcan o de las decepciones.

En este estudio, se descubrió que los alumnos deben ser enseñados, apoyados, y nutridos aun más, durante su formación profesional de enfermería, lo que beneficiaria para que los alumnos de enfermería, sean buenos pensadores críticos y reflexivos, y esto constituya a realizar una buena toma de decisiones al momento de hacer una práctica reflexiva, para que puedan desarrollar las habilidades del conocimiento, en todos los entornos que abarque la práctica de enfermería.

\section{References:}

1. Aguilera, zubirreta y castillo. (2005). Estrategias para fomentar el pensamiento crítico en estudiantes de licenciatura en enfermería.

2. Alfaro-LeFevre, R., 2003, Aplicación del proceso enfermero, 5a . ed. Barcelona: Masson, pp. 1-9, 3-4, 23.

3. Alfaro-Lefevre, R. (2007). El pensamiento crítico en enfermería. Un enfoque práctico. Barcelona: Masson.

4. Andrews M.; Guidman, J. and Humphreys, A. (1998). Reflection: does it enhance professional nursing practice? Births Journal of Nursing, 7(7), 413-417

5. Atkins, S. and Murphy, K. (1993). Reflection: a review of the literature. Journal of Advanced Nursing, 18, 1188-1192. 
6. Asociación Nacional de Universidades e Instituciones de Educación Superior, (2000): La educación superior en el siglo XXI. Líneas estratégicas de desarrollo. Una propuesta de la ANUIES, México, ANUIES

7. Baker, C. (1996). Reflective learning: a teaching strategy for critical thinking. Journal of Nursing Education, 35, 19-22.

8. Benner, P. (1987). Práctica progresiva de Enfermería. Barcelona: Grijalbo

9. Brockbank, A. and McGill, I. (2002). Aprendizaje reflexivo en la educación superior. Madrid: Morata

10. Cárdenas, Becerril, Lucila, et al., 2006, Modelo Educativo Unificado de Enfermería en México, FEMAFEE, México, 126 pp.

11. Charcnok, A. (1993). The personal tutor: a teacher's view. Nursing Standard, 7(30), 38-31

12. Clare, J. (1993a). A Challenge to the rethoric of emancipation: recreating a professional culture. Journal of Advanced Nursing, 18, 1033-1038

13. Clare, J. (1993b). Change the curriculum or transform the conditions of practice? Nurse Education Today, 13(4), 282-286

14. Clarke, M. (1986). Action and reflection: practice and theory in nursing. Journal of Advanced Nursing, 11, 3-11

15. Clarke, B., James, C. and Kelly, J. (1996). Reflective practice: reviewing the issues and refocusing the debate. International Journal of Nursing Studies, 33, 171-180

16. Cranton, P. (1994). Understanding and promoting transformative learning. S. Francisco (USA): Jossey-Bass

17. Cárdenas L. (2014). Desarrollo del Pensamiento Reflexivo y Critico en Enfermería en México 28

18. Cárdenas, Bardallo, Jiménez, Do, y Martínez (2015) desarrollo del pensamiento reflexivo y crítico en los estudiantes de enfermería de Iberoamérica. 